\title{
Fulminant Reversible Cerebral Vasoconstriction Syndrome
}

The Neurohospitalist

2018, Vol. 8(I) NP5-NP8

(C) The Author(s) 2017

Reprints and permission:

sagepub.com/journalsPermissions.nav

DOI: $10.1177 / 1941874417692923$

journals.sagepub.com/home/ $\mathrm{NHO}$

(S)AGE

\author{
Kushak Suchdev, MBBS', Gregory Norris, MD', Imad Zak, MD², \\ Wazim Mohamed, MD', and Mohammed Ibrahim, MD'
}

\begin{abstract}
Reversible cerebral vasoconstriction syndrome (RCVS) is increasingly being recognized as a diagnosis in patients presenting with thunderclap headaches. In the vast majority of the cases, the syndrome follows a benign course and patients recover completely without any significant therapeutic intervention. In the rarest of cases, RCVS follows a monophasic course with rapid worsening, severe neurological deficits, and poor outcomes. We present the case of a 25-year-old female who presented with headaches which were worsening over I week. She was admitted to the hospital and rapidly worsened to develop severe neurological deficits over the next day. Initial computed tomography scan showed areas of hemorrhage and multiple ischemic strokes. Computed tomography angiogram and a conventional cerebral angiogram both revealed multifocal vasoconstriction, highly suggestive of RCVS. Despite aggressive medical and surgical management, the patient continued to worsen and eventually died. Autopsy findings did not show evidence of vasculopathy or any other underlying disorder, further supporting the diagnosis of RCVS. The RCVS is usually a benign self-remitting condition which commonly affects young females and presents with an insidious onset of headaches. Rarely, it can have a fulminant course with devastating outcomes. This case illustrates an exceptionally uncommon clinical course of RCVS and the challenges in its treatment.
\end{abstract}

\section{Keywords}

reversible vasoconstriction syndrome, stroke in young females, secondary headache, outcomes

\section{Introduction}

Reversible cerebral vasoconstriction syndrome (RCVS) was first proposed as a distinct clinical syndrome in $2007^{1}$ to encompass uncommon and unexplained vasculopathies including Call-Fleming syndrome, ${ }^{2}$ postpartum angiopathy, ${ }^{3}$ and acute benign cerebral angiopathy. ${ }^{4}$ Most reports of RCVS describe it as a self-limiting, monophasic syndrome, presenting with sudden onset of thunderclap headache lasting for minutes to hours, often worsening over days to weeks. ${ }^{4}$ It is much more common in females than males (10:1) and the mean age of onset is 40 years. ${ }^{5}$ Several causative factors have been associated with RCVS including serotonergic and illicit drugs, pregnancy, and surgical procedures. ${ }^{2,4}$ Radiographically, it is characterized by the presence of multifocal vasoconstriction of cerebral arteries on cerebral angiography with reversibility of vasoconstriction within 12 weeks of onset. ${ }^{2,4,5}$

Given the benign monophasic nature of the disease, most patients recover completely without any long-term neurological deficits. ${ }^{4,5}$ Rarely, poor neurological outcomes and death have been reported in patients with RCVS, especially in patients who developed RCVS postpartum. ${ }^{6,7}$ We report the case of RCVS in a young woman who presented with insidious onset of headache followed by a fulminant disease course eventually resulting in her death, despite aggressive medical and surgical intervention.

\section{Case Discussion}

A 25-year-old Hispanic female with history of migraine, not on any prophylactic medications, presented to a local hospital with headache for 10 days and blurry vision. On presentation, she had mild left-sided weakness and vision loss. The vision in both eyes was limited to finger counting. Brain magnetic resonance imaging scan at that time showed multiple foci of diffusion restriction in the bilateral frontal and parietal regions as well as hemorrhage in the right parietal lobe. Computed tomography angiogram (CTA) of the head showed diffuse

\footnotetext{
' Department of Neurology, Wayne State University, Detroit, MI, USA

${ }^{2}$ Department of Radiology, Wayne State University, Detroit, MI, USA

Corresponding Author:

Kushak Suchdev, Department of Neurology, Wayne State University, 8D, UHC, 420I St Antoine, Detroit, MI, USA.

Email:ksuchdev@med.wayne.edu
} 


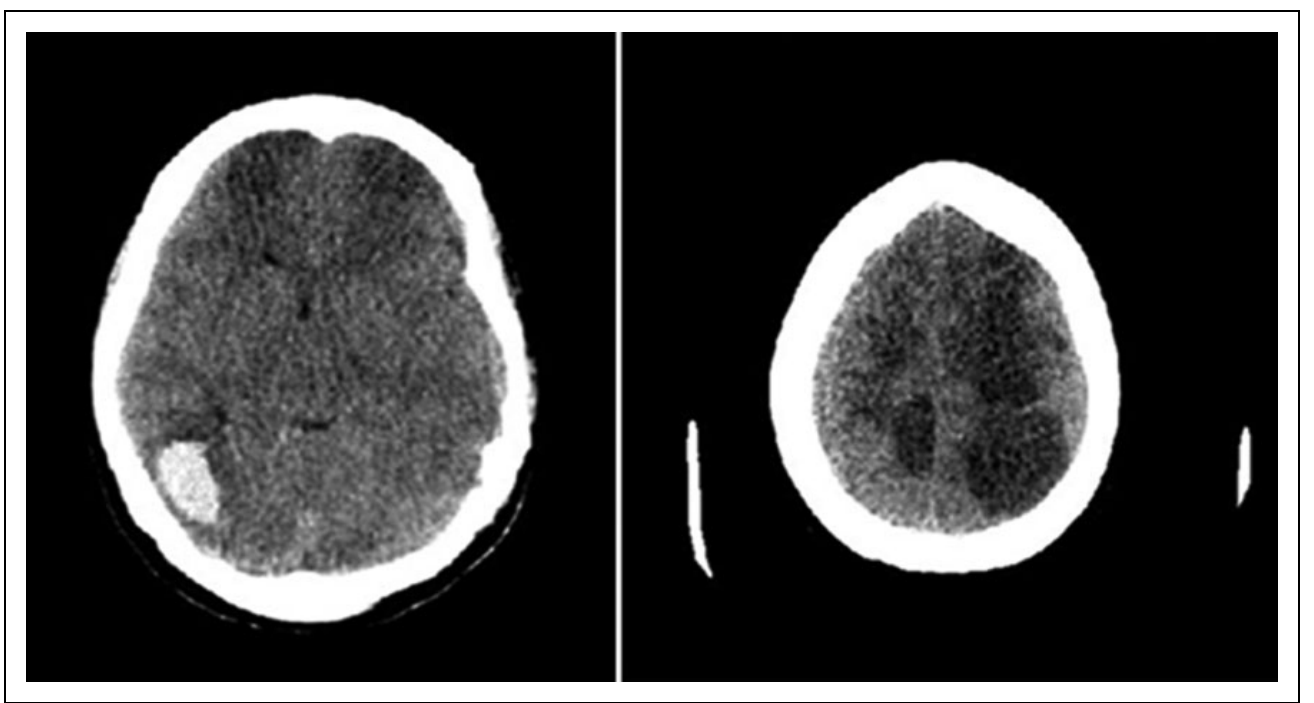

Figure I. Computed tomography (CT) scan showing right parietal hematoma and areas of ischemic stroke in the bilateral frontal, left parietal, and right parietal lobes.

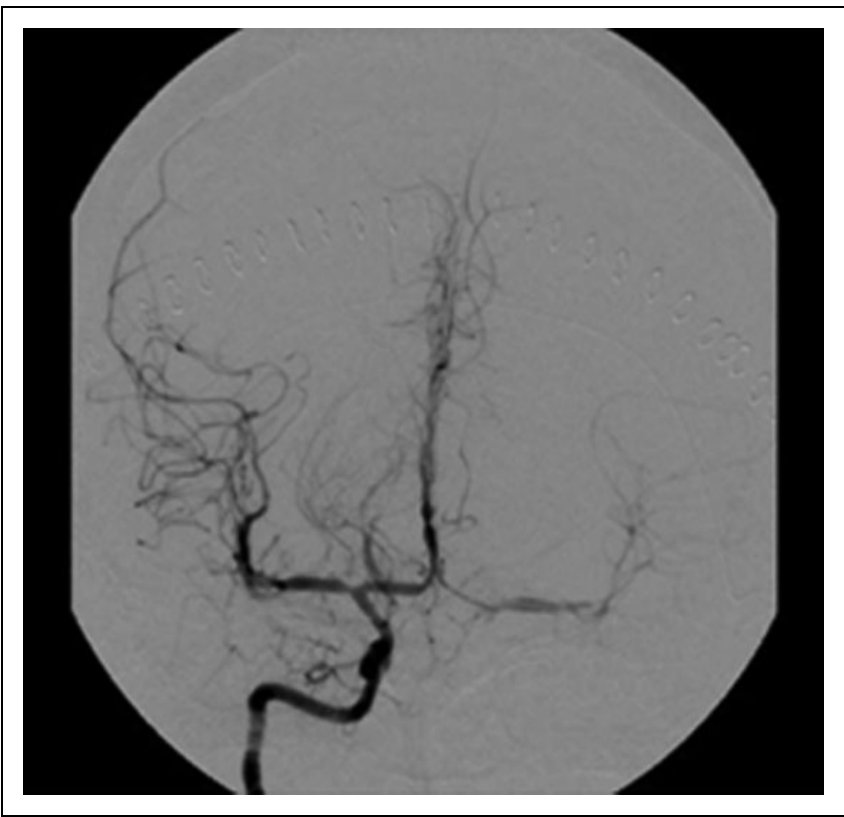

Figure 2. Cerebral angiogram showing segmental vasoconstriction and dilation in multiple vessels (arrows).

vasoconstriction of her cerebral vasculature, which was more prominent in the proximal vessels. Initial investigations including complete blood count, erythrocyte sedimentation rate, antinuclear antibodies, and extractable nuclear antigen screen were normal. Workup for pro- and hypercoagulant states including protime, partial thromboplastin time, fibrinogen, thrombin time, protein $\mathrm{C}$, protein $\mathrm{S}$, antithrombin III, lupus, factor $\mathrm{V}$ Leiden, and cardiolipin screen were negative. Toxicological screen and pregnancy test were also negative. Cerebrospinal fluid including cell count, protein, and all cultures was unremarkable. Based on these findings, a presumptive diagnosis of primary central nervous system (CNS) vasculitis was made and a high-dose intravenous methylprednisolone (IVMP) was initiated. The following day, she had a generalized seizure, was started on valproic acid, and subsequently transferred to our institution for further management.

On presentation to our hospital, the patient was awake and responsive. Neurological examination revealed equal and reactive pupils, a right field cut, vision in both eyes limited to finger counting, and left hemiparesis. She was continued on IVMP and was also started on oral nimodipine and magnesium drip to alleviate the vasospasm seen on the CTA done at the previous hospital. Next morning, she was unarousable to verbal or painful stimuli, only had withdrawal to pain in her extremities. She was emergently intubated for airway protection. Following intubation, she developed a fixed, dilated right pupil. A computed tomography (CT) scan of the head showed diffuse edematous changes with loss of gray-white matter junction and sulcal effacement without new infarcts and hemorrhage (Figure 1). The patient was treated with hypertonic saline infusion and underwent emergency decompressive hemicraniectomy. Postsurgery, a cerebral angiogram revealed diffuse vasoconstriction of cerebral vasculature, highly suggestive of RCVS (Figure 2).

An intracranial pressure (ICP) monitor was placed; propofol and fentanyl infusions were initiated to maintain her ICP below $20 \mathrm{~mm} \mathrm{Hg}$. Despite being on maximum doses of both propofol and fentanyl infusions, the patient continued to have remarkably high ICP (50-60 $\mathrm{mm} \mathrm{Hg}$ ). Hypothermia was initiated to maintain a core body temperature of $35^{\circ} \mathrm{C}$, and a pentobarbital infusion was started to decrease ICP. Even with multiple anesthetic agents and hypothermia, the ICP remained elevated and the patient did not show any improvement in her neurological status. 
Table I. Previously Reported Cases of RCVS With Poor Outcomes.

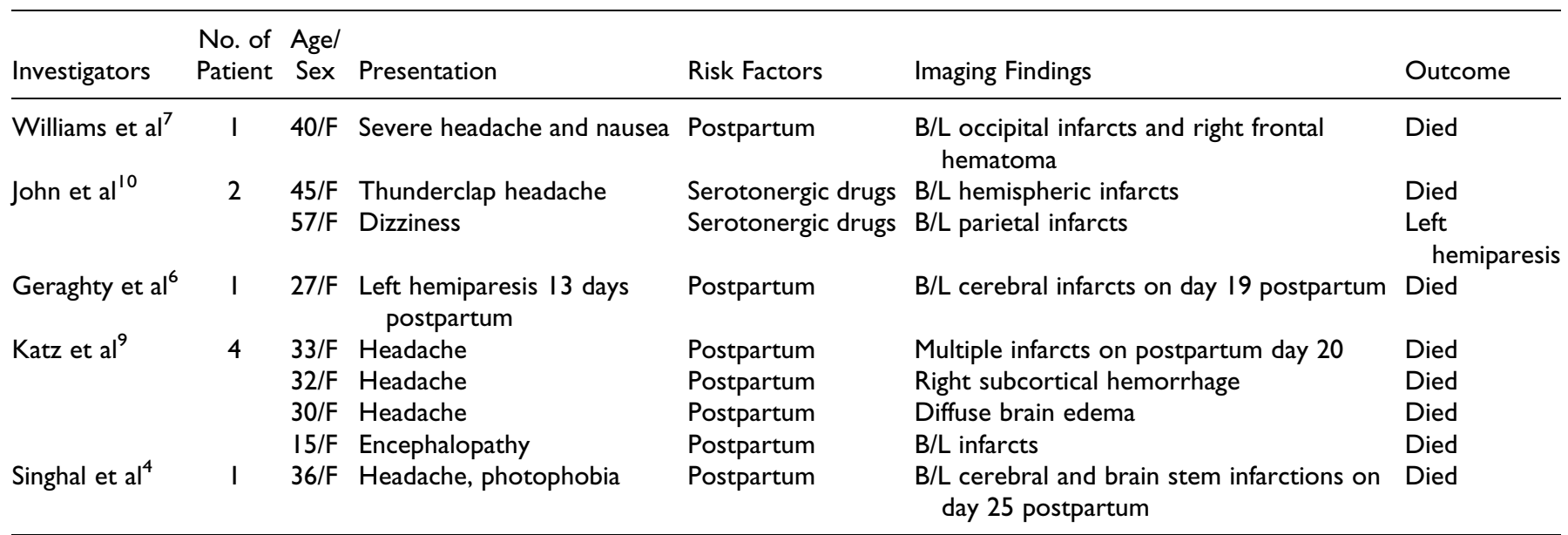

Abbreviations: B/l, bilateral; F, female; RCVS, reversible cerebral vasoconstriction syndrome.

After 48 hours of no improvement, life support was withdrawn per family's consent, and the patient died shortly thereafter. An autopsy was done which revealed diffusely swollen brain with multiple areas of infarcts and hemorrhage. No other lesions were seen. Examination of the cerebral blood vessels did not show CNS vasculitis or any other immune-mediated vasculopathy, which further supported the diagnosis of RCVS.

\section{Discussion}

Poor outcome and death due to RCVS have been rarely reported. $^{2,4,8}$ Table 1 summarizes previously reported cases of RCVS with poor outcome.

Our patient was a young female with history of migraine, a known risk factor, presenting with worsening headaches. Though she had a gradual onset of symptoms, she quickly progressed to have significant neurological deficits and became comatose.

Such rapid progression is extremely unusual in RCVS, but the findings on CTA and, later, cerebral angiogram confirmed severe vasoconstriction. These imaging findings coupled with the autopsy findings that ruled out CNS vasculitis and other vasculopathies supported the diagnosis of RCVS.

Several studies have demonstrated an association between radiological findings on the initial CT scan in patients with RCVS and permanent neurological deficits. ${ }^{3,9}$ Expectedly, patients with infarction and hemorrhage on $\mathrm{CT}$ are more likely to present with neurological deficits, but on a long-term follow-up, only a minority of these patients had permanent deficits. ${ }^{10}$ Another important predictor of long-term outcome is neurological status on admission. ${ }^{3}$ Our patient had findings of ischemic strokes and hemorrhage on her admission scan, but she presented with only mild neurological deficits. However, the rapid deterioration was extremely striking as it is not usually associated with RCVS. Thus, we believe that rapid neurological deterioration in RCVS is possibly a predictor of fulminant disease and poor outcomes.
Death due to fulminant RCVS has been reported in less than $1 \%$ of cases and is usually seen in postpartum patients or with the use of serotonergic drugs. ${ }^{6-8}$ Both of these risk factors were not present in our patient; however, the vasoconstriction as evidenced by the CTA and cerebral angiogram was progressive and irreversible leading to malignant brain edema and eventual fatal outcome. As mentioned earlier, RCVS often follows a monophasic course, and serial imaging of cerebral vessels is not indicated. Serial transcranial Doppler (TCD) has been used in limited settings in patients with RCVS to follow the degree of vasoconstriction. ${ }^{11}$ We believe that serial TCD may be especially useful in cases like ours, which present with severe or worsening neurological deficits to allow time for preemptive and potentially lifesaving measures.

Various agents have been tried for the treatment of RCVS but none of them have been proven to be effective. ${ }^{2,12,13}$ Calcium channel blockers (CCBs) are frequently used, both intra-arterially and intraorally, but whether they affect eventual outcome is still debated. ${ }^{2,12}$ Also, corticosteroids have been reported to worsen outcome in some cases but data are inconclusive. ${ }^{2,3}$ Our patient was initially started on steroids and later on oral CCBs. We are not sure whether steroids could have negatively impacted our patient. Furthermore, our patient developed fulminant brain edema which did not respond to highly aggressive surgical (hemicraniectomy) and medical (use of multiple IV anesthetics) management. This may reflect edema caused by her strokes, both ischemic and hemorrhagic, or due to the development of posterior reversible encephalopathy syndrome which often coexists with RCVS and may contribute significantly to the worsening of edema. ${ }^{14,15}$

In conclusion, our case represents an uncommon outcome of RCVS which highlights the variable clinical manifestations of this generally "benign" disorder. Even though the clinical recognition of RCVS is growing, the pathophysiology remains poorly understood. Further studies that help us understand the various unknowns of RCVS are needed to aid the development of effective therapies and eventually lead to better outcomes. 


\section{Authors' Contribution}

Kushak Suchdev developed the case report, literature search, and authored the drafts. Imad Zak supervised and coauthored the initial drafts. Wazim Mohamed helped in the preparation of drafts. Mohammed Ibrahim supervised and coauthored the drafts. Gregory Norris supervised the study creation, manuscript preparation, and finalized the drafts.

\section{Declaration of Conflicting Interests}

The authors declared the following potential conflicts of interest with respect to the research, authorship, and/or publication of this article: Gregory Norris, MD, is on the speaker board for UCB Pharmaceuticals. Informed consent was obtained from the patient's next of kin to publish this case report.

\section{Funding}

The authors received no financial support for the research, authorship, and/or publication of this article.

\section{References}

1. Calabrese LH, Dodick DW, Schwedt TJ, Singhal AB. Narrative review: reversible cerebral vasoconstriction syndromes. Ann Intern Med. 2007;146(1):34-44.

2. Mehdi A, Hajj-Ali RA. Reversible cerebral vasoconstriction syndrome: a comprehensive update. Curr Pain Headache Rep. 2014;18(9):443.

3. Robert T, Kawkabani Marchini A, Oumarou G, Uske A. Reversible cerebral vasoconstriction syndrome identification of prognostic factors. Clin Neurol Neurosurg. 2013;115(11):2351-2357.

4. Singhal AB, Hajj-Ali RA, Topcuoglu MA, et al. Reversible cerebral vasoconstriction syndromes: analysis of 139 cases. Arch Neurol. 2011;68(8):1005-1012.

5. Ducros A, Boukobza M, Porcher R, Sarov M, Valade D, Bousser MG. The clinical and radiological spectrum of reversible cerebral vasoconstriction syndrome. A prospective series of 67 patients. Brain. 2007;130(pt 12):3091-3101.

6. Geraghty JJ, Hoch DB, Robert ME, Vinters HV. Fatal puerperal cerebral vasospasm and stroke in a young woman. Neurology. 1991;41(7):1145-1147.

7. Williams TL, Lukovits TG, Harris BT, Harker Rhodes C. A fatal case of postpartum cerebral angiopathy with literature review. Arch Gynecol Obstet. 2007;275(1):67-77.

8. John S, Donnelly M, Uchino K. Catastrophic reversible cerebral vasoconstriction syndrome associated with serotonin syndrome. Headache. 2013;53(9):1482-1487.

9. Katz BS, Fugate JE, Ameriso SF, et al. Clinical worsening in reversible cerebral vasoconstriction syndrome. JAMA Neurol. 2014;71(1):68-73.

10. John S, Singhal AB, Calabrese L, et al. Long-term outcomes after reversible cerebral vasoconstriction syndrome. Cephalalgia. 2016;36(4):387-394.

11. Rubiera del Fueyo M, Molina Cateriano CA, Arenillas Lara JF, et al. Reversible segmental cerebral vasoconstriction: the value of duplex transcranial Doppler in its diagnosis and follow up [in Spanish]. Rev Neurol. 2004;38(6):530-533.

12. Zuber M, Touze E, Domigo V, Trystram D, Lamy C, Mas JL. Reversible cerebral angiopathy: efficacy of nimodipine. $J \mathrm{Neu}$ rol. 2006;253(12):1585-1588.

13. Elstner M, Linn J, Muller-Schunk S, Straube A. Reversible cerebral vasoconstriction syndrome: a complicated clinical course treated with intra-arterial application of nimodipine. Cephalalgia. 2009;29(6):677-682.

14. Dodick DW, Eross EJ, Drazkowski JF, Ingall TJ. Thunderclap headache associated with reversible vasospasm and posterior leukoencephalopathy syndrome. Cephalalgia. 2003;23(10): 994-997.

15. Singhal AB. Postpartum angiopathy with reversible posterior leukoencephalopathy. Arch Neurol. 2004;61(3):411-416. 